\title{
Combination Difference Synchronization between Identical Generalised Lotka-Volterra Chaotic Systems
}

\author{
P. Trikha, Nasreen, L. S. Jahanzaib* \\ Department of Mathematics, Jamia Millia Islamia, New Delhi, India
}

Received 25 October 2019, accepted in final revised form 6 January 2020

\begin{abstract}
This manuscript investigates the combination difference synchronization between identical Generalised Lotka-Volterra Chaotic Systems. Numerical Simulations have been performed which are in complete agreement of theoretical results.

Keywords: Combination synchronization; Difference synchronization; Generalized LotkaVolterra system.

(C) 2020 JSR Publications. ISSN: 2070-0237 (Print); 2070-0245 (Online). All rights reserved. doi: http://dx.doi.org/10.3329/jsr.v12i2.43765 J. Sci. Res. 12 (2), 183-188 (2020)
\end{abstract}

\section{Introduction}

CHAOS is one of the significant features of nonlinear phenomenon. Chaos has been described in many ways, some describe it as a disorder appearing in the events so that they appear unpredictable. Chaotic behavior exists widely in engineering, biology, economics and many other scientific disciplines. As initially chaos was not known to be so important in any field but it was only after the ice-breaking work of Pecora and Caroll who gave the concept of synchronization and synchronized two chaotic systems by designing suitable controllers. With time many synchronization schemes were developed and currently many new schemes are being developed also. Some familiar schemes used are anti-synchronization, compound synchronization, complete synchronization, combination synchronization etc. Many techniques are used to achieve the above mentioned type of synchronization like adaptive control method, active control method, tracking control method etc. [1-5].

In this article the combination difference synchronization between identical chaotic systems has been achieved. The theoretical results are verified graphically which clearly exhibit that the technique used is effective and reliable for synchronizing the considered systems. We have arranged the remaining article as: Sec 2: formulates the problem; Sec 3: develops the synchronization theory. Sec 4: conducts the combination difference synchronization scheme [6-8]. Sec 5: consists of the discussions regarding numerical simulations and displays the results performed in MATLAB. Sec 6: concludes the article.

${ }^{*}$ Corresponding author: lone.jahanzaib555@gmail.com 


\section{Problem Formulation}

The scheme of combination difference synchronization requires two chaotic drive systems and one response system. Let the one drive system be

$$
\dot{x}=f(x)
$$

and the other drive systems be

$$
\dot{y}=h(x)
$$

Let the response system be

$$
\dot{z}=r(z)+u
$$

where $x=\left(x_{1}, x_{2}, \ldots, x_{n}\right)^{T}, y=\left(y_{1}, y_{2}, \ldots, y_{n}\right)^{T}, z=\left(z_{1}, z_{2}, \ldots, z_{n}\right)^{T}$ are the state vectors of the respective systems. $f, h, r$ are three continuous vector functions. $u=\left(u_{1}, u_{2}, \ldots, u_{n}\right)^{T}: R^{n} \times R^{n} \times R^{n} \rightarrow R$ are the controllers to be designed.

Defining the error of combination difference synchronization as

$$
e=D Z-(A x-B y)
$$

Where $A=\operatorname{diag}\left(\alpha_{i}\right), B=\operatorname{diag}\left(\beta_{i}\right), D=\operatorname{diag}\left(\gamma_{i}\right)$ for $i=1,2, \ldots, n$

Definition: Systems (1)-(3) are said to be in combination difference synchronization if

$$
\lim _{t \rightarrow \infty}\|e\|=\lim _{t \rightarrow \infty}\|D Z-(A x-B y)\|=0
$$

Here, we assume $X=\operatorname{diag}\left(x_{i}\right), Y=\operatorname{diag}\left(y_{i}\right), Z=\operatorname{diag}\left(z_{i}\right)$ for $i=1,2, \ldots, n$

\section{Synchronization Theory}

We now develop the theory for combination difference synchronization among two chaotic drive systems (1)-(2) and one chaotic response system (3). We design the control function as

$$
u_{i}=\frac{\phi_{i}}{\gamma_{i}}-g_{i}-\frac{K_{i} e_{i}}{\gamma_{i}}
$$

Where $\phi_{i}=\left(\alpha_{i} f_{i}-\beta_{i} h_{i}\right)$ for $i=1,2, \ldots, n$

Theorem: To have the chaotic drive systems (1)-(2) in combination difference synchronization with (3), we choose the controllers as (4).

Proof: The error is given by:

$$
e_{i}=\gamma_{i} z_{i}-\left(\alpha_{i} x_{i}-\beta_{i} y_{i}\right) \text { for } i=1,2, \ldots, n
$$

The error dynamical system is given by:

$$
\begin{aligned}
& \dot{e}_{\imath}=\gamma_{i} \dot{z}_{i}-\left(\alpha_{i} \dot{x}_{i}-\beta_{i} \dot{y}_{i}\right) \\
&=\gamma_{i}\left(g_{i}+u_{i}\right)-\left(\alpha_{i} f_{i}-\beta_{i} h_{i}\right) \\
&=\gamma_{i}\left(g_{i}+\frac{\phi_{i}}{\gamma_{i}}-g_{i}-\frac{K_{i} e_{i}}{\gamma_{i}}-\phi_{i}\right) \\
&=\phi_{i}-K_{i} e_{i}-\phi_{i} \\
&=-K_{i} e_{i}
\end{aligned}
$$

We define the Lyapunov Function as:

$V(t)=\frac{1}{2} e^{T} e$ 


$$
\begin{aligned}
= & \frac{1}{2} \sum_{i=1}^{n} e_{i}^{2} \\
\Rightarrow V \dot{(t)} & =\sum e_{i} \dot{e}_{i} \\
& =\sum e_{i}\left(-K_{i} e_{i}\right) \\
& =-\sum K_{i} e_{i}^{2}
\end{aligned}
$$

We choose $\left(K_{1}, K_{2}, \ldots, K_{n}\right)$ in such a way such that $\left.V \dot{(t} t\right)$ is negative definite. Therefore, by Lyapunov Stability Theory, we get $\lim _{t \rightarrow \infty}\|e\|=0$. Hence, the master systems and slave system are now synchronized.

\section{Combination Difference Synchronization between Generalized Lotka Volterra Chaotic Systems}

The master system first Lotka-Volterra chaotic System given by:

$$
\begin{aligned}
& \dot{x}_{1}=x_{1}-x_{1} x_{2}+c x_{1}^{2}-a x_{1}^{2} x_{3} \\
& \dot{x}_{2}=-x_{2}+x_{1} x_{2} \\
& \dot{x}_{3}=-b x_{3}+a x_{1}^{2} x_{3}
\end{aligned}
$$

Where $x=\left(x_{1}, x_{2}, x_{3}\right)$ is the state vector of the system and $a, b, c$ its parameters. For $a=2.9851, \mathrm{~b}=3, \mathrm{c}=2$, this system shows chaotic behaviour for initial conditions $(1.2,1.2,1.2)$ displayed in Fig. 1

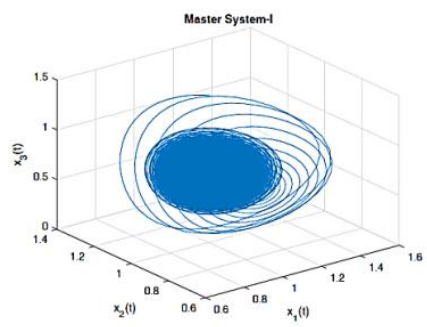

(a)

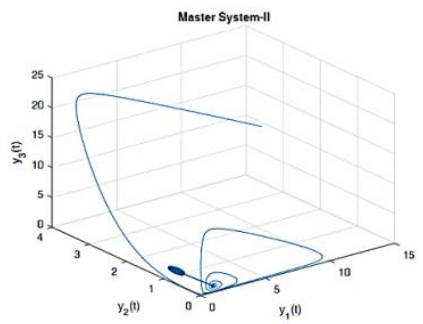

(b)

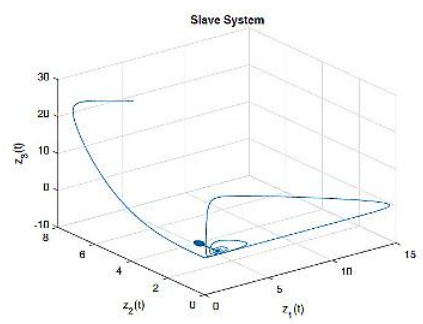

(c)

Fig. 1. (a) Phase portrait of the first master system, (b) phase portrait of the second master system and (c) phase portrait of the slave system.

The second identical master Lotka-Volterra chaotic system described respectively as follows:

$$
\begin{aligned}
& \dot{y}_{1}=y_{1}-y_{1} y_{2}+c y_{1}^{2}-a y_{1}^{2} y_{3} \\
& \dot{y}_{2}=-y_{2}+y_{1} y_{2} \\
& \dot{y}_{3}=-b y_{3}+a y_{1}^{2} y_{3}
\end{aligned}
$$

Where $y=\left(y_{1}, y_{2}, y_{3}\right)$ is the state vector of the system. For parameter values $a=$ $2.9851, \mathrm{~b}=3, \mathrm{c}=2$ and initial conditions $(14.5,3.4,10.1)$ the phase portrait shows chaotic behaviour as displayed in Fig. 1. 


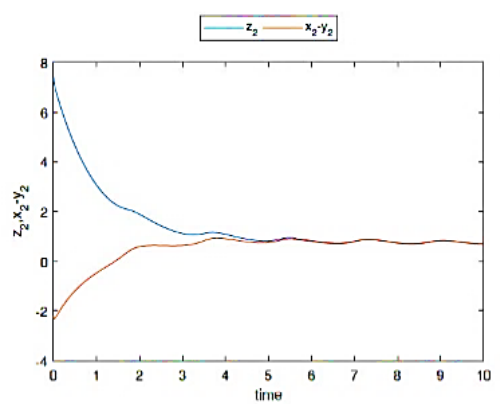

(a)

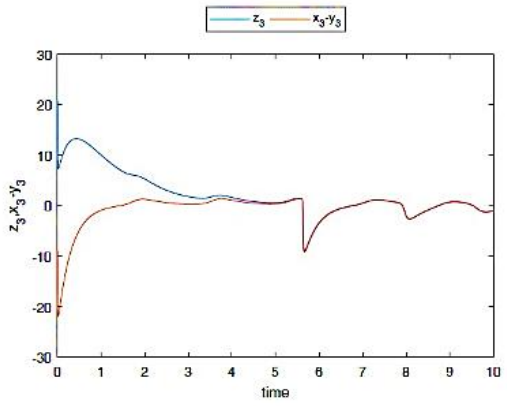

(c)

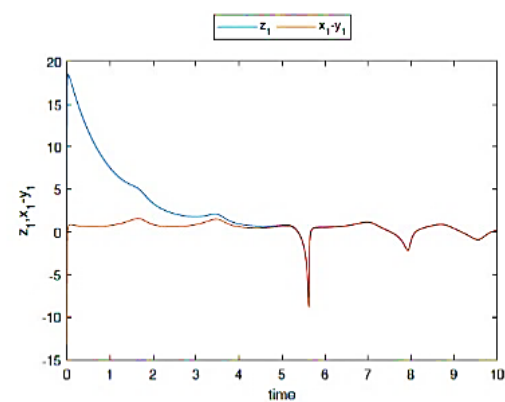

(b)

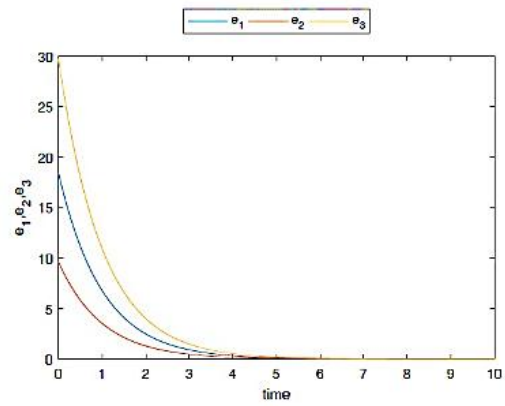

(d)

Fig 2. The synchronized trajectories and the simultaneous error plot.

The slave system is described by the identical chaotic Lotka-Volterra System described as:

$$
\begin{aligned}
& \dot{z}_{1}=z_{1}-z_{1} z_{2}+c z_{1}^{2}-a z_{1}^{2} z_{3}+u_{1} \\
& \dot{z}_{2}=-z_{2}+z_{1} z_{2}+u_{2} \\
& \dot{z}_{3}=-b z_{3}+a z_{1}^{2} z_{3}+u_{3}
\end{aligned}
$$

Where $z=\left(z_{1}, z_{2}, z_{3}\right)$ is the state vector of the system. For parameter values $a=$ $2.9851, \mathrm{~b}=3, \mathrm{c}=2$ and initial conditions $(5.1,7.4,20.8)$ the system shows chaotic behavior and $u_{1}, u_{2}, u_{3}$ are the controllers.

We define the error $\left(e_{1}, e_{2}, e_{3}\right)$ as:

$$
\begin{aligned}
& e_{1}=\gamma_{1} z_{1}-\left(\alpha_{1} x_{1}-\beta_{1} y_{1}\right) \\
& e_{2}=\gamma_{2} z_{2}-\left(\alpha_{2} x_{2}-\beta_{2} y_{2}\right) \\
& e_{3}=\gamma_{3} z_{3}-\left(\alpha_{3} x_{3}-\beta_{3} y_{3}\right)
\end{aligned}
$$

On substituting the values of the derivatives and simplifying, we get:

$$
\begin{gathered}
\dot{e}_{1}=\gamma_{1}\left(z_{1}-z_{1} z_{2}+c z_{1}^{2}-a z_{1}^{2} z_{3}+u_{1}\right)-\left(\alpha_{1}\left(x_{1}-x_{1} x_{2}+c x_{1}^{2}-a x_{1}^{2} x_{3}\right)-\beta_{1}\left(y_{1}\right.\right. \\
\left.\left.\quad-y_{1} y_{2}+c y_{1}^{2}-a y_{1}^{2} y_{3}\right)\right) \\
\dot{e}_{2}=\gamma_{2}\left(-z_{2}+z_{1} z_{2}+u_{2}\right)-\left(\alpha_{2}\left(-x_{2}+x_{1} x_{2}\right)-\beta_{2}\left(-y_{2}+y_{1} y_{2}\right)\right) \\
\dot{e}_{3}=\gamma_{3}\left(-b z_{3}+a z_{1}^{2} z_{3}+u_{3}\right)-\left(\alpha_{3}\left(-b x_{3}+a x_{1}^{2} x_{3}\right)-\beta_{3}\left(-b y_{3}+a y_{1}^{2} y_{3}\right)\right)
\end{gathered}
$$

We choose the control functions: 


$$
u_{1}=\frac{\phi_{1}}{\gamma_{1}}-g_{1}-\frac{K_{1} e_{1}}{\gamma_{1}}
$$

Where $\phi_{1}=\left(\alpha_{1} f_{1}-\beta_{1} h_{1}\right)$

$$
u_{2}=\frac{\phi_{2}}{\gamma_{2}}-g_{2}-\frac{K_{2} e_{2}}{\gamma_{2}}
$$

Where $\phi_{2}=\left(\alpha_{2} f_{2}-\beta_{2} h_{2}\right)$

$$
u_{3}=\frac{\phi_{3}}{\gamma_{3}}-g_{3}-\frac{K_{3} e_{3}}{\gamma_{3}}
$$

Where $\phi_{3}=\left(\alpha_{3} f_{3}-\beta_{3} h_{3}\right)$

Substituting (11) into (10), we get

$$
\begin{aligned}
& \dot{e_{1}}=-K_{1} e_{1} \\
& \dot{e_{2}}=-K_{2} e_{2} \\
& \dot{e_{3}}=-K_{3} e_{3}
\end{aligned}
$$

Consider the Lyapunov function as $V(e(t))=\frac{1}{2} e(t) e(t)^{T}$

$$
\begin{gathered}
=\frac{1}{2}\left(e_{1}^{2}+e_{2}^{2}+e_{3}^{2}\right) \\
V(\dot{e}(t))=e_{1} \dot{e}_{1}+e_{2} \dot{e}_{2}+e_{3} \dot{e}_{3} \\
=e_{1}\left(-K_{1} e_{1}\right)+e_{2}\left(-K_{2} e_{2}\right)+e_{3}\left(-K_{3} e_{3}\right) \\
=-K_{1} e_{1}^{2}-K_{2} e_{2}^{2}-K_{3} e_{3}^{2}
\end{gathered}
$$

Where $K_{1}, K_{2}, K_{3}>0$ are constants.

$\Rightarrow V(\dot{e}(t))$ is negative definite.

Therefore by Lyapunov Stability Theory, we know that error tends to zero, i.e. $e_{i} \rightarrow 0$ for $i=1,2,3$ implying complete synchronization has been attained.

\section{Numerical Simulations and Discussions}

We have carried out the numerical simulations using MATLAB. We have considered here, $\alpha_{i}=\beta_{i}=\gamma_{i}=\delta_{i}=1$, for $i=1,2,3$. Also we have considered $K_{i}=1$ for $i=$ $1,2,3$. Fig. 2 displays the synchronized trajectories of systems (6)-(8) with system (9). Fig. 2 also displays the simultaneous error plot tending to zero with time.

\section{Conclusion}

In this paper, combination difference synchronization has been performed on identical chaotic Generalized Lotka-Volterra Systems. This type of synchronization technique can be used to determine the effect of some coexisting species on some particular species represented by slave system of Generalized Lotka-Volterra model. Further, the theoretical results are in excellent agreement with computational results. 


\section{References}

1. A. Khan and M. A. Bhat, Math. Methods Appl. Sci. 40, 5654 (2017). https://doi.org/10.1002/mma.4416

2. A. Khan, D. Khattar, and N. Prajapati. Int. J. Dynamics Control 1 (2017).

3. A. Khan and P. Trikha, SN Appl. Sci. 1, 757 (2019). https://doi.org/10.1007/s42452-019-0776$\underline{\mathrm{X}}$

4. A. Khan and A.Tyagi, Pramana, 90, 67 (2018). https://doi.org/10.1007/s12043-018-1555-8

5. A. Khan, D. Khattar and N. Prajapati, arXiv:1804.03028v1 (2018).

6. L. M. Pecora and T. L. Carroll, Phys. Rev. Lett. 64, 821 (1990). https://doi.org/10.1103/PhysRevLett.64.821

7. E. D. Dongmo, K. S.Ojo, P. Woafo, and A. N. Njah, J. Comput. Nonlinear Dynamics 13, Article ID 051005 (2018). https://doi.org/10.1115/1.4039626

8. J. Sun, Y. Shen, and G. Cui, Adv. Math. Phys. 2015 (2015). https://doi.org/10.1155/2015/921515 